\title{
AGROMETEOROLOGIA
}

\section{SIMULAÇÃO DO DESENVOLVIMENTO DA BATATA CULTIVAR ASTERIX EM CINCO CENÁRIOS DE MUDANÇAS CLIMÁTICAS EM SANTA MARIA, RS $\left(^{1}\right)$}

\author{
NEREU AUGUSTO STRECK $\left({ }^{2, *}\right)$; ISABEL LAGO $\left({ }^{3}\right)$; CLEBER MAUS ALBERTO $\left({ }^{4}\right)$; \\ DILSON ANTÔNIO BISOGNIN $\left({ }^{2}\right)$
}

\begin{abstract}
RESUMO
Em estudos do impacto de mudanças climáticas sobre agroecossistemas, um aspecto importante a quantificar é o efeito do aquecimento global sobre o desenvolvimento vegetal. O objetivo desse trabalho foi simular o impacto de cenários climáticos de aumento de temperatura do ar no desenvolvimento da batata (Solanum tuberosum L.), em Santa Maria, (RS). Cenários meteorológicos de mudanças climáticas $\left(+2{ }^{\circ} \mathrm{C},+3{ }^{\circ} \mathrm{C},+4{ }^{\circ} \mathrm{C},+5{ }^{\circ} \mathrm{C}\right.$ e $\left.+6{ }^{\circ} \mathrm{C}\right)$ para os próximos cem anos foram gerados com Weather Generator LARS-WG usando-se como base a série de dados meteorológicos observados de 1969 a 2003, em Santa Maria, RS. Por meio de um modelo matemático, calculou-se a taxa diária de desenvolvimento da planta e a data dos principais estágios de desenvolvimento da batata, cv. Asterix: emergência, início de tuberização, início de senescência e colheita. Foram consideradas duas épocas de cultivo da batata, o cultivo de outono (plantio em 10/2) e o cultivo de primavera (plantio em 20/7). Verificou-se, para o cultivo de outono, um grande aumento (até 42 dias) no ciclo total (emergência-colheita) com o aumento da temperatura do ar. Já para o cultivo de primavera, obteve-se uma tendência de um pequeno encurtamento (5-8 dias) do ciclo total da batata com o aumento da temperatura média do ar, dependendo do nível de aquecimento. Fases intermediárias do desenvolvimento da batata poderão ter alterações que dependem da época de cultivo. Essas diferentes tendências no comportamento do desenvolvimento da batata refletem a complexidade da resposta dos agroecossistemas à mudança climática.
\end{abstract}

Palavras-chave: Aquecimento global, risco climático, previsão climática, fenologia.

\section{ABSTRACT \\ SIMULATION OF POTATO CULTIVAR ASTERIX DEVELOPMENT UNDER FIVE CLIMATE CHANGE SCENARIOS IN SANTA MARIA}

In studies of the impact of climate change on agroecosystems, it is important to quantify the effect of global warming on crop development. The objective of this study was to simulate the impact of increasing air temperature scenarios on the potato (Solanum tuberosum L.) development in Santa Maria,

$\left({ }^{1}\right)$ Recebido para publicação em 26 de outubro de 2005 e aceito em 11 de julho de 2006.

$\left({ }^{2}\right)$ Departamento de Fitotecnia, Centro de Ciências Rurais (CCR), Universidade Federal de Santa Maria (UFSM), 97105-900 Santa Maria (RS), Brasil. E-mail: nstreck1@smail.ufsm.br. ${ }^{*}$ Autor para correspondência.

$\left({ }^{3}\right)$ Aluna do Curso de Agronomia, CCR, UFSM. Bolsista de Iniciação Científica da Fundação de Amparo à Pesquisa do Rio Grande do Sul (FAPERGS).

$\left({ }^{4}\right)$ Aluno do Programa de Pós-Graduação em Engenharia Agrícola, CCR, UFSM, Santa Maria (RS). Bolsista da Fundação de Coordenação de Aperfeiçoamento de Pessoal de Nível Superior (CAPES). 
Rio Grande do Sul State. Climate change scenarios of increasing air temperature $\left(+2{ }^{\circ} \mathrm{C},+3{ }^{\circ} \mathrm{C},+4{ }^{\circ} \mathrm{C},+5\right.$ ${ }^{\circ} \mathrm{C}$, and $+6{ }^{\circ} \mathrm{C}$ ) for the next 100 years were created with the LARS-WG Weather Generator using the 19692003 database in Santa Maria, RS, Brazil. Using a simulation model, the daily development rate and the date of the following development stages were calculated: emergence, tuber initiation, beginning of plant senescence, and crop harvest. Two potato planting dates were assumed, a Fall planting (10/02) and a Spring planting (20/07). An increase in air temperature led to a large increase (up to 42 days) and to a small decrease (5-8 days) in the duration of the emergence-harvest phase for the Fall and Spring plantings, respectively, depending upon the increase in air temperature. Intermediate developmental phases may be altered depending upon the growing season. The trends of potato development in different scenarios reflect the complexity of agroecossystems response to climate change.

Key words: Global warming, climate risk, climate forecast, phenology.

\section{INTRODUÇÃO}

Parece existir consenso geral na comunidade científica de que o clima terrestre está mudando (IPCC, 2001; WeIss et al., 2003; KERR, 2005). A concentração de $\mathrm{CO}_{2}$ da atmosfera terrestre está aumentando, a uma taxa de $0,4 \%-0,5 \%$ ano $^{-1}$ (IPCC, 1995 ; SOMBROEK e GOMES, 1996), o que corresponde ao aumento de $1,0-1,8 \mu \mathrm{mol}$ $\mathrm{CO}_{2}$ mol- $^{1}$ ano- $^{1}$ (IPCC, 1995; Unsworth e HogsetT, 1996). Como resultado do aumento da concentração de $\mathrm{CO}_{2}$ atmosférico, a temperatura média do ar deverá aumentar devido ao aumento do efeito estufa na atmosfera terrestre, fenômeno freqüentemente referido como aquecimento global (ROSENBER et al., 1983; IPCC, 1995). Estudos indicam um aumento de 1 a $6{ }^{\circ} \mathrm{C}$ na temperatura média do ar até o fim desse século em vários locais do Planeta (IPCC, 1995; MAHLMAN, 1997; IPCC, 2001), incluindo o Brasil (SiqueIRA et al., 1994, 2000, 2001; Assad et al., 2004).

Em plantas, o aumento da concentração de $\mathrm{CO}_{2}$ atmosférico causa aumento da taxa de crescimento, pois o $\mathrm{CO}_{2}$ é o substrato primário para fotossíntese (ROSENBERG et al., 1983; TAIZ e ZEIGER, 2004). Plantas com metabolismo $C 3$ são mais beneficiadas pelo aumento da concentração de $\mathrm{CO}_{2}$ atmosférico do que plantas com metabolismo $\mathrm{C} 4$ (Tubiello et al., 2000; Siqueira et al., 2001; Streck, 2005). No entanto, se o aumento da concentração de $\mathrm{CO}_{2}$ for acompanhado de aumento da temperatura do ar, poderá não haver aumento no crescimento e produtividade das culturas devido ao encurtamento do ciclo (Wiegand e Cuellar, 1981; Sionit et al., 1987; Hunt et al., 1991; Butterfield e Morison, 1992; SHAYKEWICH, 1995, SiqueIRA et al., 2001) e aumento da respiração (fotorespiração e fase escura da respiração) (ROSENBERG et al., 1983; LONG, 1991; Vu et al., 1997; TAIZ e Zeiger, 2004). Portanto, em estudos de impactos das mudanças climáticas sobre os agroecossistemas, devese dar especial atenção ao efeito do aumento de temperatura (aquecimento global) no desenvolvimento vegetal e assim caracterizar mudanças no ciclo das culturas em diferentes cenários meteorológicos.
Esses estudos são difíceis de serem realizados experimentalmente, devido à limitação de mão-de-obra e recursos financeiros (ANDERSEN et al., 2001). Dessa forma, os modelos matemáticos são ferramentas que permitem descrever as interações entre a planta/ cultura e o ambiente, e podem ser usados em estudos de mudanças climáticas em agroecossistemas. Assim, modelos de simulação do desenvolvimento de plantas podem ajudar a entender e quantificar como o ciclo das culturas seria afetado pelo aquecimento global.

A batata (Solanum tuberosum L.), família Solanaceae, ocupa o quarto lugar em quantidade de produção de alimentos, sendo superada apenas pelo trigo, arroz e milho (HAWKES, 1994; NYENDE et al., 2005). A produção anual mundial de batata é em torno de 320 milhões de toneladas (FAO, 2005), sendo cultivada em aproximadamente 125 países e mais de um bilhão de pessoas consomem batata diariamente em todo o mundo (Mullins et al., 2006). A batata é a principal cultura olerícola no Brasil em área e em preferência alimentar, com uma área cultivada de aproximadamente $150 \mathrm{mil}$ ha/ano e produtividade média em torno de $20 \mathrm{t} / \mathrm{ha}$ (LOPES e Buso, 1997; IBGE, 2004).

A temperatura e o fotoperíodo são os dois principais fatores abióticos que influenciam o desenvolvimento da cultura da batata (Levy e KeDAR, 1985). A batata é uma planta de dia curto para início de tuberização, e de dia longo e/ou neutra para florescimento, ou seja, dias curtos aceleram o início de tuberização e dias longos aceleram o florescimento (LEVY e KEDAR, 1985). Esses fatores são representados em modelos matemáticos de simulação do desenvolvimento das culturas por funções de respostas à temperatura e fotoperíodo (STRECK et al., 2003).

Estudos do impacto das mudanças climáticas na agricultura do Brasil têm aumentado nos últimos anos, e estimativas de rendimento de várias culturas agrícolas obtidas em diversas regiões brasileiras diante de cenários de mudança climática já foram relatadas (Siqueira et al., 1994, 2000, 2001; Assad et 
al., 2004). No entanto, não se constatou na literatura qualquer trabalho que indicasse o impacto da mudança climática no ciclo da cultura da batata em condições brasileiras, o que constituiu o incentivo para esse esforço científico. O objetivo desse trabalho foi simular o impacto de cenários climáticos de aumento de temperatura do ar no desenvolvimento da batata em Santa Maria, RS.

\section{MATERIAL E MÉTODOS}

Esse estudo foi realizado nas condições de Santa Maria, RS (latitude: $29^{\circ} 43^{\prime} \mathrm{S}$, longitude: $53^{\circ} 43^{\prime} \mathrm{W}$ e altitude: $95 \mathrm{~m}$ ). O clima da região, segundo a classificação de Köppen, é Cfa, ou seja, subtropical úmido, sem estação seca definida, com verões quentes (Moreno, 1961). Dados meteorológicos de 1969 até 2003 coletados na Estação Climatológica Principal pertencente ao $8 .^{\circ}$ Distrito de Meteorologia/Instituto Nacional de Meteorologia, localizada no Departamento de Fitotecnia da Universidade Federal de Santa Maria, Santa Maria, RS foram usados nesse estudo.

Os cenários meteorológicos de mudança climática foram gerados com o Weather Generator LARS-WG (SEMENOV et al., 1998), tendo-se como base a série de dados meteorológicos diários observados de 1969 a 2003. Com base nesse conjunto de dados foram gerados os cenários com aumento de $+2{ }^{\circ} \mathrm{C},+3$ ${ }^{\circ} \mathrm{C},+4{ }^{\circ} \mathrm{C},+5{ }^{\circ} \mathrm{C}$ e $+6{ }^{\circ} \mathrm{C}$ na temperatura média diária do ar, mantendo-se a mesma amplitude térmica diária da base de dados originais, até o fim de um período de cem anos. O LARS-WG é um modelo estocástico que tem sido usado em estudos do impacto de cenários de mudança climática sobre o desempenho de agroecossistemas (Weiss et al., 2003; Richter e Semenov, 2005; Streck e Alberto, 2006). Esse modelo estocástico pode gerar séries meteorológicas (duração superior a 30 anos podem ser consideradas séries climáticas) por meio de distribuições de probabilidade semi-empíricas que mantém as estatísticas da série original; as estatísticas das séries climáticas geradas devem ser comparadas com as estatísticas da série de dados meteorológicos observados (SEMENOv e BARrow, 1997; Semenov et al., 1998). Para Santa Maria, RS, a comparação entre séries climáticas com cenários de mudança climática com a série de dados meteorológicos observados de 1969 a 2003 foi realizada anteriormente (STRECK e AlbeRT, 2006). Constatou-se que não há diferença entre as estatísticas, e que as séries climáticas geradas pelo LARS-WG podem ser usadas nesse estudo. Foi, então, utilizado um modelo de simulação de desenvolvimento para reproduzir o efeito dessas mudanças climáticas sobre o desenvolvimento da batata em duas datas de plantio em cada ano nesse período de cem anos.

O modelo de simulação do desenvolvimento da batata WANG e ENGEL (1998), usado nesse estudo, foi anteriormente calibrado e avaliado por PAULA (2005). Nesse modelo, o ciclo da cultura da batata é dividido em três fases: vegetativa, que vai da emergência (EM) ao início da tuberização (IT); tuberização, do IT ao início da senescência (IS); e senescência, do IS à colheita (CO). Primeiramente o modelo calcula a taxa diária de desenvolvimento da planta (r) a partir da EM. Em seguida, o estágio de desenvolvimento (DS) em que a planta se encontra é calculado acumulando-se os valores da taxa diária de desenvolvimento, ou seja, DS $=\sum$ r. O DS é 0 na EM, 1 no IT, 1,8 no IS e 2 na CO. Optou-se em dividir o ciclo de desenvolvimento da batata e atribuir os números $0,1,1,8$ e 2 para os estágios de desenvolvimento acima, com base na proposta de dividir o ciclo de desenvolvimento de outras culturas anuais dessa maneira (PenNing de VRIes et al., 1989; Goudriann e VAN LAAR, 1994; StReCK et al., 2003). A forma geral do modelo de WANG e ENGEL para batata é:

para a fase vegetativa (EM - IT),

$r=\operatorname{rmax}, v f(T) f(P)$

para a fase de tuberização (IT - IS),

$r=\operatorname{rmax}, t \mathrm{f}(\mathrm{T})$

e para a fase de senescência (IS - CO),

$r=\operatorname{rmax}, s f(T)$

em que: $r$ é a taxa diária de desenvolvimento $\left(\right.$ dia $\left.^{-1}\right)$; rmax, $\mathrm{v}, \operatorname{rmax}, \mathrm{t}$ e rmax,s são as taxas máximas de desenvolvimento diária $\left(\mathrm{dia}^{-1}\right)$ na fase vegetativa, de tuberização e de senescência respectivamente, e $f(T)$ e $f(P)$ são funções de resposta à temperatura e ao fotoperíodo, variando de 0 a 1 respectivamente.

A função de resposta à temperatura $\mathrm{f}(\mathrm{T})$ é uma função beta definida como (WANG e EENGEL, 1998; STRECK, 2002; STRECK et al., 2003):

$\mathrm{f}(\mathrm{T})=\left[2\left(\mathrm{~T}-\mathrm{T}_{\min }\right)^{\alpha}\left(\mathrm{T}_{\mathrm{opt}}-\mathrm{T}_{\min }\right)^{\alpha}-\left(\mathrm{T}-\mathrm{T}_{\min }\right)^{2 \alpha}\right] /\left(\mathrm{T}_{\mathrm{opt}}-\mathrm{T}_{\min }\right)^{2 \alpha}$

$\alpha=\ln 2 / \ln \left[\left(\mathrm{T}_{\max }-\mathrm{T}_{\min }\right) /\left(\mathrm{T}_{\mathrm{opt}}-\mathrm{T}_{\min }\right)\right]$

Se $T<$ Tmin ou $T>$ Tmax, então $f(T)=0$

em que: $T_{\min }, T_{\text {opt }}$ e $T_{\max }$ são as temperaturas cardinais de desenvolvimento e $\mathrm{T}$ é a temperatura média diária. Na fase vegetativa, $\mathrm{T}_{\min }$ foi definida como $4{ }^{\circ} \mathrm{C}$ (Hartz e Moore, 1978) e Topt e Tmax como 15 e $28^{\circ} \mathrm{C}$ respectivamente (Ingram e Mccloud, 1984). $\mathrm{Na}$ fase de tuberização as temperaturas cardinais $\left(\mathrm{T}_{\min }, \mathrm{T}_{\text {opt }}\right.$ e $\left.\mathrm{T}_{\max }\right)$ foram, respectivamente, 7,21 e $30^{\circ} \mathrm{C}$ (SANDS et al., 1979; Manrique e Hodges, 1989). 
As temperaturas cardinais de desenvolvimento durante a fase de senescência foram as mesmas da primeira fase (EM-IT), ou seja, Tb $=4$ ${ }^{\circ} \mathrm{C}$, Topt $=15^{\circ} \mathrm{C}$ e $\operatorname{Tmax}=28^{\circ} \mathrm{C}$ (Paula, 2005; Paula et al., 2005).

A função de resposta ao fotoperíodo f(P) é uma função exponencial negativa para uma planta de dia curto (Paula, 2005):

$f(P)=\exp [-\omega(P-P c)]$

Se $\mathrm{P}<\mathrm{Pc}$, então $\mathrm{f}(\mathrm{P})=1$

sendo: $\omega$ o coeficiente de sensibilidade ao fotoperíodo (h-1), que depende da cultivar, $\mathrm{P}$, o fotoperíodo (h) e Pc, o fotoperíodo crítico (h), que, para uma planta de dia curto, é o fotoperíodo abaixo do qual a resposta é máxima. $\mathrm{O}$ fotoperíodo $(\mathrm{P})$, incluindo o crepúsculo civil (quando o sol está entre 0 a $6^{\circ}$ abaixo do plano do horizonte), foi calculado com o algoritmo de KiesLing (1982).

A cultivar de batata Asterix foi selecionada por ser bastante cultivada no Estado do Rio Grande do Sul. Asterix é uma cultivar de ciclo médio, tubérculo com epiderme rosada e polpa amarelo-clara, duplo propósito (consumo de mesa e processamento) e longa dormência (NivAA, 1997). A estimativa dos valores dos coeficientes $\operatorname{rmax}, \mathrm{v}, \operatorname{rmax}, \mathrm{t}, \operatorname{rmax}, \mathrm{s}, \omega$ e Pc foi com o procedimento NLIN do SAS usando-se o método de Marquardt, que minimiza a soma de quadrados do erro da estimativa. Para a estimativa desses coeficientes foram utilizados dados diários de temperatura e fotoperíodo e as datas de EM, IT, IS e $\mathrm{CO}$ de seis épocas de plantio da batata Asterix em Santa Maria, RS (28/2/2003, 27/3/2003, 12/8/2003, 15/9/2003, 15/10/2003 e 20/11/2003) descritos em Paula (2005) e Paula et al. (2005). Para essa cultivar, os valores estimados de $r_{\max , v}, r_{\max , t}, r_{\max , s}$, foram $0,0793,0,0260$ e $0,012074 \mathrm{dia}^{-1}$ respectivamente (Paula, 2005). O valor estimado de Pc foi 10,7 h e o valor estimado de $\omega$ foi 0,0645 h-1 (PAula, 2005). O modelo de desenvolvimento foi então avaliado com dados independentes provenientes de nove épocas de plantio da batata Asterix em Santa Maria (21/1/2003, $12 / 2 / 2003,24 / 12 / 2003,28 / 1 / 2004,27 / 2 / 2004,26 /$ 3/2004, 26/4/2004, 31/5/2004 e 26/8/2004), com desempenho satisfatório em todas as épocas (PAula, 2005) e, portanto, apropriado para esse estudo.

Para a simulação do desenvolvimento da batata nos cenários climáticos foram consideradas duas datas de plantio, 10 de fevereiro e 20 de julho, as quais estão dentro das épocas recomendadas para os cultivos de outono e primavera dessa cultura, respectivamente, na região de Santa Maria (BISOGNIN, 1996). O dia da emergência, considerado como o primeiro dia da simulação do desenvolvimento da batata, foi considerado 10 dias após o plantio dos tubérculos no plantio de outono e 20 dias no plantio de primavera, conforme dados médios acumulados de experimentos em Santa Maria (D.A. Bisognin, comunicação pessoal). A duração, em dias, das fases EM-IT, IT-IS e IS-CO foram calculadas para os cem anos em todos os cenários climáticos para ambos os cultivos de outono e primavera.

A análise dos dados constou da análise de regressão linear $(y=a+b x)$ entre duração das fases de desenvolvimento (dias) e anos em cada cenário climático para cada época de cultivo (outono e primavera). O coeficiente angular da regressão (b) representa a modificação (aumento ou diminuição) na duração de cada fase de desenvolvimento por ano (dia/ano) em cada cenário climático. Usou-se o teste $\mathrm{t}$ para testar as hipóteses $\mathrm{H}_{0}$ (coeficiente $\mathrm{b}$ não difere de zero) e $\mathrm{H}_{1}$ (coeficiente $\mathrm{b}$ difere de zero). Com os coeficientes $b$ que diferiram de zero a $5 \%$ e $1 \%$ de probabilidade de erro calculou-se a mudança percentual na duração das fases de desenvolvimento no fim de cem anos de cada cenário climático por:

Mudança $\%=\left\{\left[\left(\left(b^{*} 100\right)+\mathrm{DM}_{\mathrm{o}}\right) / \mathrm{DM}_{\mathrm{o}}\right]^{* 100\}-100}\right.$

em que: b é o valor do coeficiente angular da regressão (dias ano $^{-1}$ ) e DMo, a duração média (dias) da fase de desenvolvimento no cenário sem mudança climática.

Foram também calculados os desvios (diferença entre os cenários com mudança e o cenário sem mudança climática) da duração (dias) das fases durante os últimos 10 anos de cada cenário meteorológico. Desvio positivo significa que a duração da fase de desenvolvimento aumenta em cenários de mudanças climáticas, enquanto desvio negativo significa que a duração da fase de desenvolvimento diminui em cenários de mudanças climáticas.

\section{RESULTADOS E DISCUSSÃO}

A duração, em dias, das diferentes fases do ciclo de desenvolvimento da batata cv. Asterix ao longo dos cem anos dos cenários sem aumento e com aumento de $6{ }^{\circ} \mathrm{C}$ na temperatura do ar estão na figura 1. A duração das fases nos outros cenários de aumento de temperatura foi intermediária entre os dados das duas curvas apresentadas em cada painel da figura 1 .

O coeficiente angular (b) da regressão linear entre a duração das diferentes fases de desenvolvimento e os anos, não foi significativamente diferente de zero pelo teste $t$ para o cenário sem mudança climática nas duas épocas de cultivo (Tabela 1). Esses resultados significam que não há tendência significativa de incremento ou diminuição da duração das fases de 
desenvolvimento durante os cem anos do cenário sem mudança climática, resultado de nenhuma tendência de aumento ou diminuição da temperatura do ar na base de dados e na série sintética criada pelo LARSWG para esses cenários. Qualquer mudança na duração das fases nos outros cenários é devido ao aumento de temperatura dos cenários de aquecimento global. A duração média das fases EM-IT, IT-IS, IS-CO e EM-CO no cenário sem mudança climática foram, respectivamente, $29,34,26$ e 88 dias no cultivo de outono e 14, 39, 23 e 76 dias no cultivo de primavera.
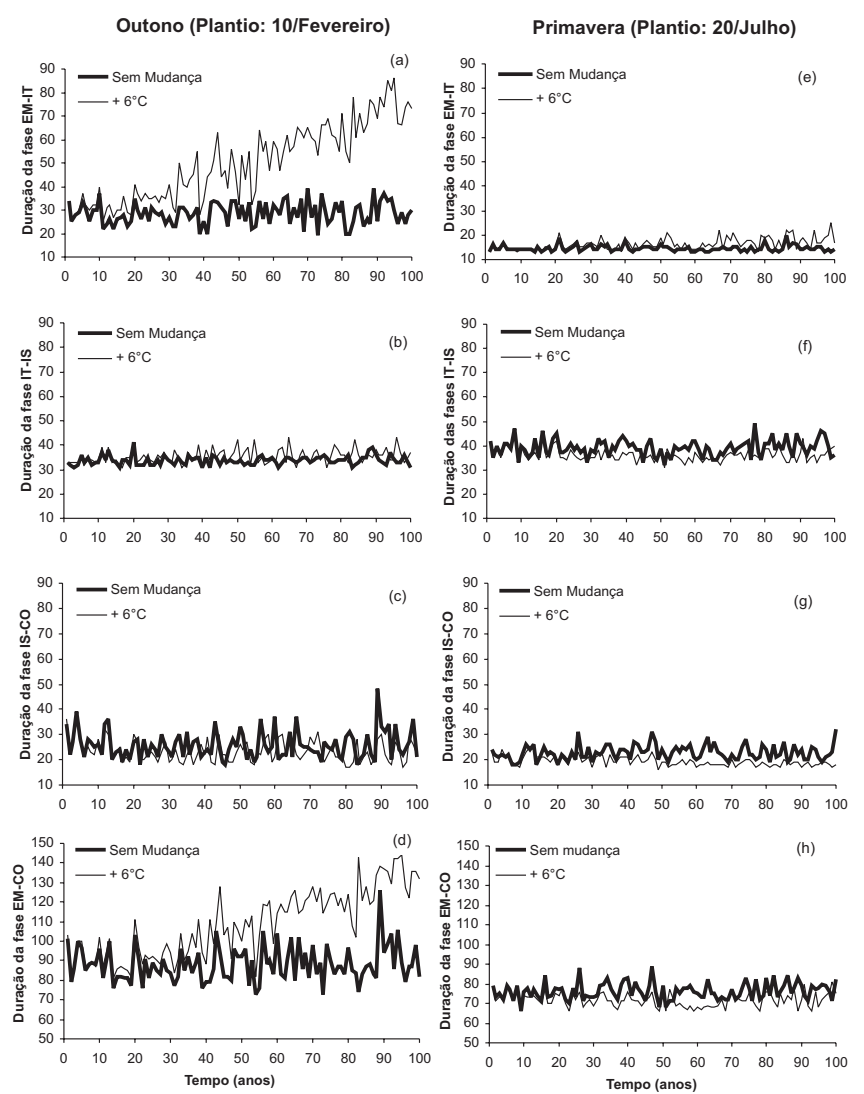

Figura 1. Duração (dias) simulada das fases de desenvolvimento EM-IT, IT-IS, IS-CO e EM-CO da batata, $\mathrm{cv}$. Asterix, para o cultivo na primavera e outono, em cenário sem mudança climática e com aumento de $6{ }^{\circ} \mathrm{C}$ na temperatura média diária do ar no fim de cem anos. Santa Maria, RS. EM = emergência, IT = início de tuberização, IS = início de senescência, $\mathrm{CO}=$ colheita.

Nos cenários com mudança climática, o coeficiente angular (b) da regressão linear foi não significativo apenas na fase IS-CO nos cenários $+2{ }^{\circ} \mathrm{C}$, $+3{ }^{\circ} \mathrm{C},+4{ }^{\circ} \mathrm{C}$ e $+5{ }^{\circ} \mathrm{C}$ para o cultivo de outono e nas fases EM-IT nos cenários $+2{ }^{\circ} \mathrm{C} \mathrm{e}+3{ }^{\circ} \mathrm{C}$ e EM-CO no cenário $+6{ }^{\circ} \mathrm{C}$ para o cultivo de primavera. Em todas as outras situações, o coeficiente angular foi significativamente diferente de zero, indicando efeito da mudança climática sobre a duração das fases de desenvolvimento da batata cultivar Asterix.
Tabela 1. Valores do coeficiente angular da regressão linear entre a duração das fases de desenvolvimento e os anos, e mudança percentual na duração das fases de desenvolvimento da batata cultivar Asterix no fim de cem anos nos cenários climáticos para Santa Maria, RS

\begin{tabular}{lccc}
\hline $\begin{array}{l}\text { Fase de } \\
\text { desenvolvimento }\end{array}$ & $\begin{array}{c}\text { Cenário } \\
\left(\text { dias } \text { ano }^{-1}\right)\end{array}$ & $\begin{array}{c}\text { Coeficiente } \\
\text { angular }\end{array}$ & $\begin{array}{c}\text { Mudança na } \\
\text { duração } \\
\text { da fase }(\%)\end{array}$ \\
\hline
\end{tabular}

Cultivo de outono

(Plantio: 10/fevereiro)

EM-IT

IT-IS

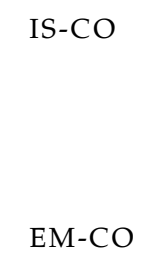

\begin{tabular}{cc} 
Sem mudança & $0,0152 \mathrm{~ns}$ \\
$+2{ }^{\circ} \mathrm{C}$ & $0,1417^{* *}$ \\
$+3{ }^{\circ} \mathrm{C}$ & $0,2285^{* *}$ \\
$+4{ }^{\circ} \mathrm{C}$ & $0,3220^{* *}$ \\
$+5{ }^{\circ} \mathrm{C}$ & $0,4252^{* *}$ \\
$+6{ }^{\circ} \mathrm{C}$ & $0,5084^{* *}$ \\
Sem mudança & $0,0096^{\mathrm{ns}}$ \\
$+2{ }^{\circ} \mathrm{C}$ & $0,0221^{* *}$ \\
$+3{ }^{\circ} \mathrm{C}$ & $0,0249^{* *}$ \\
$+4{ }^{\circ} \mathrm{C}$ & $0,0289^{* *}$ \\
$+5{ }^{\circ} \mathrm{C}$ & $0,0231^{* *}$ \\
$+6{ }^{\circ} \mathrm{C}$ & $0,0254^{* *}$ \\
Sem mudança & $0,0081^{\mathrm{ns}}$ \\
$+2{ }^{\circ} \mathrm{C}$ & $0,00744^{\mathrm{ns}}$ \\
$+3{ }^{\circ} \mathrm{C}$ & $0,0035^{\mathrm{ns}}$ \\
$+4{ }^{\circ} \mathrm{C}$ & $-0,00744^{\mathrm{ns}}$ \\
$+5{ }^{\circ} \mathrm{C}$ & $-0,01511^{\mathrm{ns}}$ \\
$+6{ }^{\circ} \mathrm{C}$ & $-0,0268^{*}$ \\
Sem mudança & $0,0329^{\text {ns }}$ \\
$+2{ }^{\circ} \mathrm{C}$ & $0,1712^{* *}$ \\
$+3{ }^{\circ} \mathrm{C}$ & $0,2569^{* *}$ \\
$+4{ }^{\circ} \mathrm{C}$ & $0,3435^{* *}$ \\
$+5{ }^{\circ} \mathrm{C}$ & $0,4332^{* *}$ \\
$+6{ }^{\circ} \mathrm{C}$ & $0,5071^{* *}$ \\
\multicolumn{4}{c}{$\mathrm{Cultivo} \mathrm{de} \mathrm{primavera}$} \\
(Plantio: $20 /$ julho $)$
\end{tabular}

EM-IT

IT-IS
Sem mudança
$+2{ }^{\circ} \mathrm{C}$
$+3{ }^{\circ} \mathrm{C}$
$+3{ }^{\circ} \mathrm{C}$
$+5{ }^{\circ} \mathrm{C}$
$+6{ }^{\circ} \mathrm{C}$

Sem mudança

$+2{ }^{\circ} \mathrm{C}$

$+3{ }^{\circ} \mathrm{C}$

$+4{ }^{\circ} \mathrm{C}$

$+5{ }^{\circ} \mathrm{C}$

$+6{ }^{\circ} \mathrm{C}$

IS-CO

Sem mudança

$+2{ }^{\circ} \mathrm{C}$

$+3{ }^{\circ} \mathrm{C}$

$+4{ }^{\circ} \mathrm{C}$

$+5{ }^{\circ} \mathrm{C}$

$+6{ }^{\circ} \mathrm{C}$

EM-CO

Sem mudança

$+2{ }^{\circ} \mathrm{C}$

$+3{ }^{\circ} \mathrm{C}$

$+4{ }^{\circ} \mathrm{C}$

$+5{ }^{\circ} \mathrm{C}$

$+6{ }^{\circ} \mathrm{C}$

49,7

80,2

113,9

149,2

178,4

6,5

7,4

8,6

6,9

7,5

$-$

$-$

-

$-10,4$

19,4

29,2

39,0

49,2

57,6

(Plantio: 20/julho)

$-0,0003^{\text {ns }}$

$0,0009^{\mathrm{ns}}$

$0,0146^{*}$

$0,0292^{* *}$

$0,0461^{* *}$

$0,0092^{\text {ns }}$

$-0,0229^{*}$

$-0,0307^{* *}$

$-0,0349^{* *}$

$-0,0363^{* *}$

$-0,0184^{*}$

$0,0106^{\mathrm{ns}}$

$-0,0179^{*}$

$-0,0298^{* *}$

$-0,0312^{* *}$

$-0,0366^{* *}$

$-0,0346^{* *}$

$0,0195^{\mathrm{ns}}$

$-0,0399^{* *}$

$-0,0542^{* *}$

$-0,0515^{* *}$

$-0,0437^{* *}$

$-0,0068^{\mathrm{ns}}$

10,1

20,3

32,0

$-5,8$

$-7,8$

$-8,9$

$-9,3$

$-4,7$

$-7,9$

$-13,1$

$-13,7$

$-16,1$

$-15,2$

$-$

$-5,2$

$-7,1$

$-6,7$

$-5,7$

ns=não significativo, ${ }^{*}=$ significativo a $5 \%,{ }^{* *}=$ significativo a $1 \%$. $\mathrm{EM}=$ emergência, IT=início de tuberização, IS=início de senescencia e $\mathrm{CO}=$ colheita. 
O aumento de temperatura do ar resultou em um alongamento da fase EM-IT em ambas as épocas de cultivo; para o cultivo de outono, essa tendência de aumento da duração da fase EM-IT foi muito maior do que para o cultivo de primavera (Tabela 1, Figura 1a e 1e). No fim dos cem anos de cada cenário, a estimativa de aumento na duração dessa fase variou de $49,7 \%$ a $178,4 \%$ para o cultivo de outono e $10,1 \%$ a $32,0 \%$ para o cultivo de primavera, proporcionalmente ao aumento de temperatura do ar nos cenários. Para a fase IT-IS, no fim dos cem anos houve tendência de aumento da duração dessa fase de $6,5 \%$ a $8,6 \%$ no cultivo de outono e diminuição de $5,8 \%$ a $9,3 \%$ no cultivo de primavera (Tabela 1, Figura 1b e 1f). A duração da fase IS-CO, quando foi afetada pelo aumento da temperatura, diminuiu com elevação de temperatura do ar nas duas épocas de cultivo, sendo a redução no fim dos cem anos de $10,4 \%$ a $+6{ }^{\circ} \mathrm{C}$ no cultivo de outono e de $7,9 \%$ a $15,2 \%$ proporcional ao aumento de temperatura no cultivo de primavera no fim dos cem anos (Tabela 1, Figura 1c e 1g).

Para o ciclo total (EM-CO), no fim dos cem anos nos cenários, houve um grande aumento na duração $(19,4 \%$ a $57,6 \%)$ com aumento da temperatura do ar no cultivo de outono; para o cultivo de primavera, houve uma pequena tendência de encurtamento no ciclo total $(5,2 \%$ a $7,1 \%)$ com aumento da temperatura do ar no fim dos cem anos nos cenários (Tabela 1, Figura 1d e 1h). Note-se que o aumento na duração do ciclo total da cultura no cultivo de outono foi devido ao grande aumento da duração da fase de EM-IT com o aumento na temperatura do ar (Tabela 1, Figura 1).

Considerando os últimos dez anos de cada cenário, no cultivo de outono (Figura 2a) houve um grande aumento na duração das fases EM-IT e EMCO nos cenários com aumento de temperatura do ar, alcançando por volta de 45 e 43 dias, respectivamente, no cenário de $+6^{\circ} \mathrm{C}$. Observou-se, também nessa época de cultivo, pequeno aumento (inferior a 5 dias) na duração da fase IT-IS em todos os cenários e diminuição, até de 6 dias na fase IS$\mathrm{CO}$ que foi significativa apenas para o cenário $+6^{\circ} \mathrm{C}$. Já no cultivo de primavera (Figura $2 b$ ), houve um pequeno encurtamento das fases IT-IS, IS-CO e ciclo total (EM-CO), o qual foi mais expressivo (5-8 dias) nos cenários com aumento de $+3{ }^{\circ} \mathrm{C},+4{ }^{\circ} \mathrm{C}$ e $+5{ }^{\circ} \mathrm{C}$ na temperatura média do ar, decrescendo no cenário de $+6{ }^{\circ} \mathrm{C}$ a ponto de não ser significativo o encurtamento do ciclo total nesse último cenário $(+6$ $\left.{ }^{\circ} \mathrm{C}\right)$. Já a duração da fase EM-IT aumentou proporcionalmente à elevação da temperatura do ar, alcançando 5 dias no cenário de $+6{ }^{\circ} \mathrm{C}$.
Outono (Plantio: 10/Fevereiro)
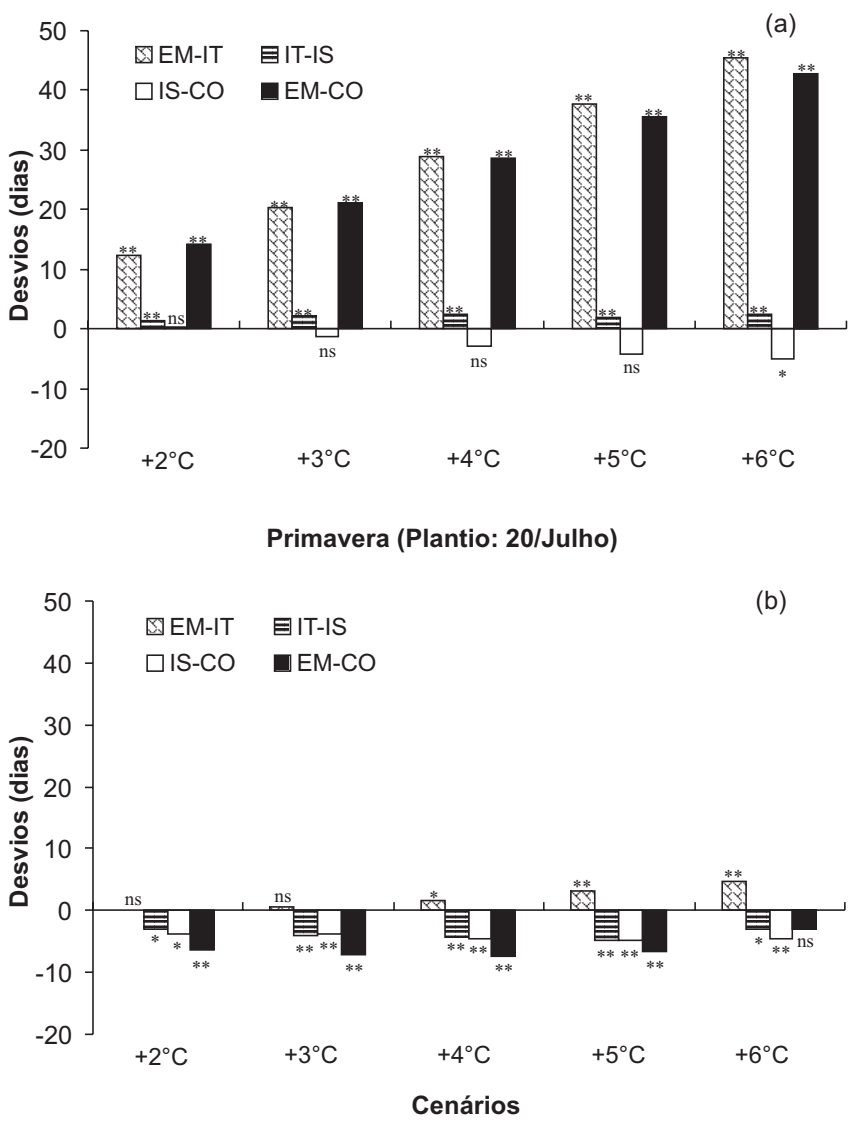

Figura 2. Desvios (em dias) na duração das fases de desenvolvimento EM-IT, IT-IS, IS-CO, EM-CO da batata, $\mathrm{cv}$. Asterix, simulados em diferentes cenários climáticos com aumento de temperatura $\left(+2{ }^{\circ} \mathrm{C},+3{ }^{\circ} \mathrm{C},+4{ }^{\circ} \mathrm{C},+5\right.$ ${ }^{\circ} \mathrm{C}$ e $+6{ }^{\circ} \mathrm{C}$ ) em relação ao cenário sem mudança climática para os cultivos de outono (a) e primavera (b), Santa Maria, RS. As barras representam a média dos últimos 10 anos de cada cenário climático. EM = emergência, IT = início de tuberização, IS = início de senescência, $\mathrm{CO}=$ colheita. ns=não significativo, ${ }^{*}=$ significativo a $5 \%,{ }^{* *}=$ significativo a $1 \%$.

Os resultados de aumento na duração de fases de desenvolvimento da cultura com o aumento de temperatura do ar obtidos nesse estudo são muito mais expressivos do que o encurtamento das fases com o aumento de temperatura (Figura 2). Esses resultados são diferentes de outros na literatura, que relatam uma redução do ciclo com o aumento da temperatura do ar (ButTerfield e Morison, 1992; Siqueira et al., 1994, 2000; STRECK, 2005).

O encurtamento do ciclo das culturas simulado em diferentes cenários de mudança climática (aumento de temperatura) relatado na literatura ocorre, na maioria dos casos, devido ao fato de que a simulação do desenvolvimento é baseada no conceito da soma térmica, que acumula os graus-dia acima de uma temperatura-base e sem um limite superior de 
temperatura (WIEGAND e CUELLAR, 1981; BUTTERFIELD e Morison, 1992; MCMAster e Wilhelm, 1997). Esse acúmulo de graus-dia, no entanto, não é biologicamente realístico, pois a resposta do desenvolvimento vegetal, como qualquer outro processo biológico, é uma função das três temperaturas cardinais, ou seja, a temperatura mínima, a ótima e a máxima (Streck, 2002; Streck et al., 2003; Streck, 2004). A taxa de desenvolvimento da cultura aumenta com a temperatura na faixa entre a temperatura mínima e a temperatura ótima, e decresce quando a temperatura é superior à temperatura ótima, chegando a zero para valores de temperatura igual ou superior à temperatura máxima de desenvolvimento (ShaykeWich, 1995; Streck, 2002; Streck, 2004). Nesse estudo, o desenvolvimento da batata não foi simulado com base no conceito da soma térmica, e sim com um modelo multiplicativo que tem uma função de resposta à temperatura $[\mathrm{f}(\mathrm{T})]$ com as três temperaturas cardinais de desenvolvimento (WANG e ENGEL, 1998).

Outra característica importante do modelo de desenvolvimento de WANG e ENGEL (1998) usado nesse estudo é que a resposta do desenvolvimento da batata à temperatura do ar é descrito por uma função nãolinear. Tem sido demonstrado que a resposta do desenvolvimento vegetal à temperatura é linear apenas em uma faixa entre a temperatura mínima e a temperatura ótima (STRECK, 2002; STRECK et al., 2003; STRECK, 2004). Em cenários de aumento de temperatura, espera-se o aumento da freqüência de valores além da temperatura ótima, onde a resposta do desenvolvimento da cultura é não-linear. Aqui novamente o método da soma térmica, o qual usa a pressuposição de uma resposta linear do desenvolvimento vegetal à temperatura, está aberto a críticas, o que é mais um motivo para interpretar os resultados de estudos numéricos que relatam a duração das fases do ciclo de desenvolvimento em cenários de aquecimento global utilizando a soma térmica (Wiegand e Cuellar, 1981; Butterfield e Morison, 1992; SiqueIRA et al., 1994, 2000, 2001) com cautela.

Na fase EM-IT houve aumento de duração com a elevação da temperatura nas duas épocas de cultivo. Como o aumento na duração da fase EM-IT foi pequeno no cultivo de primavera, não houve aumento no ciclo total (EM-CO) nessa época, pois ocorreu encurtamento nas outras duas fases (Figura $2 b$ ). Já no cultivo de outono, o enorme acréscimo na duração da fase EM-IT reflete em grande aumento no ciclo total (Figura 1a). Analisando-se os valores de temperatura nos primeiros 60 dias de cada cultivo, verifica-se que as temperaturas nos cenários com mudança climática foram bem maiores que a temperatura ótima $\left(15^{\circ} \mathrm{C}\right)$ e mais próximos, inclusive superiores, da temperatura máxima $\left(28^{\circ} \mathrm{C}\right)$, assumidas no modelo de desenvolvimento para a fase de EM-IT, no cultivo de outono do que no cultivo de primavera (Figura 3). Assim, os valores de $f(T)$ nessa fase foram sempre menores nos cenários de mudança climática comparado com o cenário sem mudança, especialmente no cultivo de outono (inclusive sendo zero em alguns dias), retardando o desenvolvimento. Portanto, o grande alongamento do ciclo total de desenvolvimento da batata com o aumento na temperatura do ar projetado para o cultivo de outono explica-se pela grande freqüência de dias com temperaturas supra-ótimas durante a fase EM-IT nessa época de cultivo. As características e o fato de que o modelo de WANG e ENGEL (1998) ter sido avaliado com dados independentes provenientes de várias épocas de plantio da batata, inclusive fora da época recomendada (durante o verão) em Santa Maria (PAULA, 2005), sugerem que as estimativas de aumento da fase EM-IT e do ciclo total (EM-CO) no cultivo de outono (Figura 2a) e no cultivo de primavera (Figura 2 b) são realísticas.
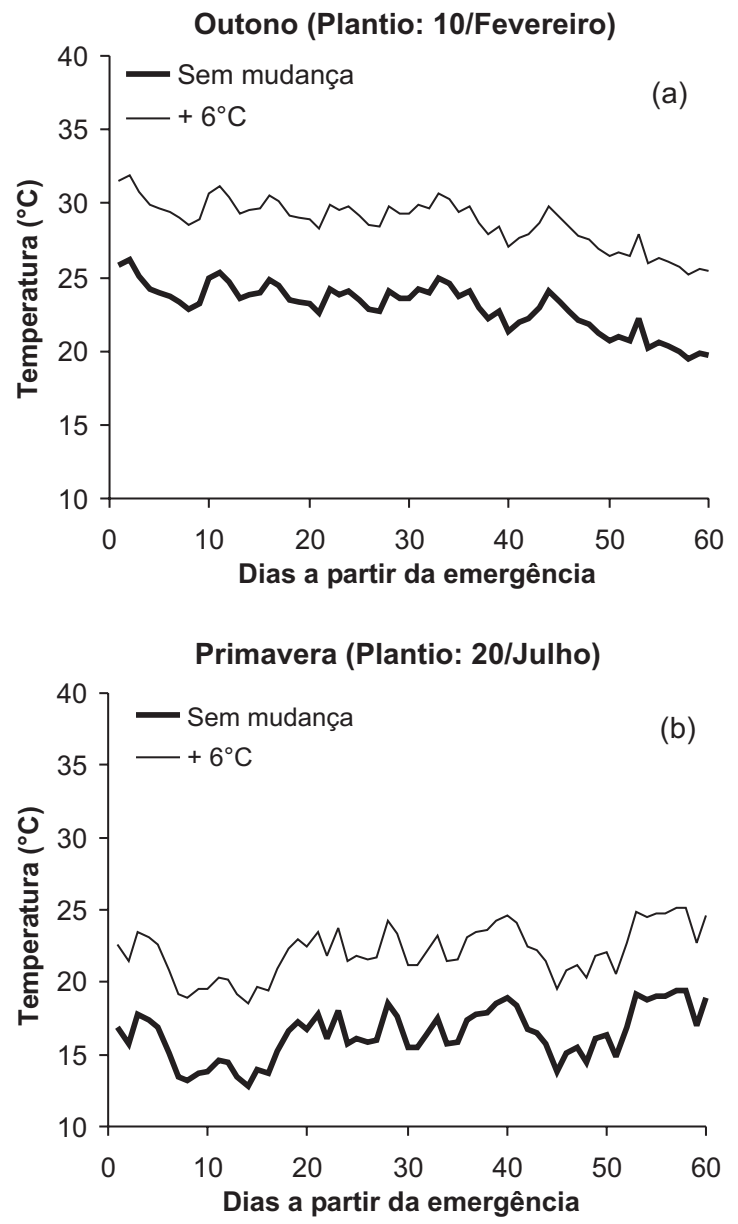

Figura 3. Temperatura média diária nos primeiros 60 dias a partir da emergência da batata, cv. Asterix, para o cultivo de outono (a) e primavera (b) em cenário sem mudança climática e com aumento de $6{ }^{\circ} \mathrm{C}$ na temperatura média diária do ar. Os valores são a média dos últimos 10 anos dos cenários. Santa Maria, RS. 
Modificações na duração das fases de desenvolvimento da batata em cenários de mudanças climáticas podem ter conseqüências em vários outros processos fisiológicos e no manejo da cultura de batata. Por exemplo, a fase EM-IT se caracteriza pelo estabelecimento do sistema radicular e da área foliar. Caso o aquecimento global se confirmar, essa fase aumentaria em Santa Maria, especialmente no outono (Figura 2), necessitando que a planta se torne mais resistente ao déficit hídrico (cultivares novas), pois o período de estabelecimento radicular seria mais longo e a área foliar aumentaria. Uma área foliar maior seria benéfica, pois a planta de batata poderia iniciar a tuberização com mais capacidade fotossintética. No que se refere ao manejo da cultura da batata, um alongamento da fase EM-IT mudaria a data de aplicação do nitrogênio em cobertura, a qual é recomendada no IT (Westermann e KleinKope, 1985; Lopes e Buso, 1997), especialmente no cultivo de outono.

A fase intermediária (IT-IS), que no cenário sem mudança climática foi de aproximadamente 35 dias no cultivo de outono (Figura 1b) e de aproximadamente 40 dias no cultivo de primavera (Figura 1f), poderia aumentar até três dias no cultivo de outono e diminuir em até cinco dias no cultivo de primavera se a temperatura aumentar em até $6{ }^{\circ} \mathrm{C}$ (Figura 2). Nessa fase, os fotoassimilados são usados, principalmente, para o crescimento dos tubérculos na extremidade dos estolões. Assim, seria esperado que o cultivo de outono fosse mais beneficiado pelo aquecimento global do que o de primavera, pois a duração dessa fase tem relação direta com o rendimento de tubérculos (SouzA, 2003). Na fase IS$\mathrm{CO}$, os fotoassimilados são translocados para os tubérculos, elevando o teor de matéria seca ao máximo. Essa fase teve duração de aproximadamente 25 dias no cultivo de outono (Figura 1c) e de aproximadamente 20 dias na primavera (Figura 1g) no cenário sem mudança climática; em ambas as épocas haveria uma redução de aproximadamente cinco dias para cenários com aumentos de $4{ }^{\circ} \mathrm{C}$ ou mais na temperatura média do ar. Essa redução na fase de IS-CO certamente tem efeitos negativos no rendimento de tubérculos, pois a duração dessa fase também tem relação direta com esse rendimento (SouzA, 2003).

Com relação ao ciclo total (EM-CO), a duração dessa fase, que no cenário sem mudança climática no cultivo de outono foi entre 70 e 100 dias (Figura 1d), pode aumentar em até 40 dias no fim dos cem anos no cenário de $+6{ }^{\circ} \mathrm{C}$ (Figura 2a). No cultivo de primavera, essa fase teve duração de 70 a 80 dias no cenário sem mudança (Figura 1h) e pode diminuir entre 5 e 8 dias em cenários de aumento de temperatura (Figura 2b). Um aumento na duração do ciclo total com o aumento de temperatura projetado para o cultivo de outono pode ter impacto negativo na qualidade dos tubérculos colhidos, pois a colheita sendo atrasada ocorreria em condições meteorológicas adversas no inverno que elevam a umidade do solo.

Por fim, associado aos efeitos do aquecimento global sobre o desenvolvimento vegetal, cabe ressaltar que o aumento na temperatura tem efeito negativo de aumentar a respiração do tecido vegetal em qualquer fase do ciclo de desenvolvimento, o que tende a anular os efeitos benéficos do aumento na duração das fases e o aumento de $\mathrm{CO}_{2}$, especialmente em plantas C3 (STRECK, 2005) como a batata.

Pelos resultados desse estudo, constata-se que a resposta do desenvolvimento da batata às mudanças climáticas são complexas e que as possíveis mudanças climáticas, principalmente o aquecimento global, podem aumentar os riscos do agronegócio da batata. A fragilidade dos agroecossistemas diante de possíveis alterações atmosféricas no futuro exigirá mudanças nas recomendações para as culturas, caso as projeções de aumento de temperatura se confirmarem. O impacto dessas mudanças pode ser grande para o produtor. Por exemplo, o zoneamento agrícola poderá ser alterado, tornando muitas áreas inaptas ao cultivo de determinadas culturas agrícolas e dificultando o crédito agrícola, como demonstrado por AssAD et al. (2004) para a cultura do café.

\section{CONCLUSÕES}

1. O aumento de temperatura do ar de $2{ }^{\circ} \mathrm{C}$ é suficiente para modificar a duração de ao menos algumas fases de desenvolvimento da batata cultivar Asterix em Santa Maria, RS.

2. No cultivo de outono da batata cultivar Asterix são projetados grandes aumentos na duração da fase EM-IT e ciclo total (EM-CO) com o aumento de temperatura do ar, enquanto no cultivo de primavera o efeito do aquecimento global é menor, com projeções de acréscimo na duração da fase EMIT e diminuição no ciclo total (EM-CO).

3. Para a duração das fases intermediárias (ITIS e IS-CO) da batata cultivar Asterix, as projeções diante do aumento de temperatura dependem da fase e da época de cultivo. A fase IT-IS é projetada para se elevar com o aumento de temperatura no cultivo de outono e diminuir no cultivo de primavera. Já a fase IS-CO é projetada para diminuir em ambas as épocas de cultivo, mas no outono apenas se a temperatura se elevar $6^{\circ} \mathrm{C}$. 


\section{REFERÊNCIAS}

ANDRESEN, J.A.; ALAGARSWAMY, G.; ROTZ, C.A.; RITCHIE, J.T.; LEBARON, A.W. Weather impacts on maize, soybean, and alfalfa production in the Great Lakes Region, 1895-1996. Agronomy Journal, Madison, v.93, p.1059-1070, 2001.

ASSAD, E.D.; PINTO, H.S.; ZULLOJUNIOR, J.; ÁVILA, A.M.H. Impacto das mudanças climáticas no zoneamento agroclimático do café no Brasil. Pesquisa Agropecuária Brasileira, Brasília, v.39, n.11, p.1057-1064, 2004.

BISOGNIN, D.A. Recomendações técnicas para o cultivo da batata no Rio Grande do Sul e Santa Catarina. Santa Maria: UFSM, 1996. 64p.

BUTTERFIELD, R.E.; MORISON, J.I.L. Modeling the impact of climate warming on winter cereal development. Agricultural and Forest Meteorology, Amsterdam, v.62, n.3, p.241-261, 1992.

FAO. FAOSTAT data 2005. Disponível em http:// faostat.fao.org/faostat. Acesso em 25 de julho de 2005.

GOUDRIAAN, J.; VAN LAAR, H.H. Modeling potencial crop growth processes. Dordrecht: Kluwer, 1994. 238p.

HARTZ, T.K.; MORE, F.D. Prediction of potato yield using temperature and insolation data. American Potato Journal, Orono, v.55, n.7, p.431-436, 1978.

HAWKES, J.G. Origins of cultivated potatoes and species relationships. In: BRADSHAW, J.E.; MACKAY, R.R. Potato genetics. Cambridge: CAB International, 1994. p.3-42.

HUNT, L.A.; VAN DER POORTEN, G.; PARARAJASINGHAM, S. Postanthesis temperature effects on duration and rate of grain filling in some winter and spring wheats. Canadian Journal of Plant Science, Ottawa, v.71, n.5, p.609-617, 1991.

IBGE, 2004. Levantamento sistemático da produção agrícola confronto das safras 2003 e estimativas para 2004. Disponível em http://www.ibge.com.br. Acesso em 22 de novembro de 2004.

INGRAM, K.T.; McCLOUD, D.E. Simulation of potato growth and development. Crop Science, Madison, v.24,n.1, p.21-27, 1984.

IPCC [Intergovernmental Panel on Climate Change]. Climate Change: the IPCC Scientific Assesment. Cambridge: University Press, 1995. 288p.

IPCC [Intergovernmental Panel on Climate Change]. Climate change 2001: the scientific bases - The contribution of the working group I of the third assessment report. Cambridge: University Press, 2001. 944p.

KERR, R.A. Millennium hottest decade retains its title, for now. Science, Washington, v.307, p.828-829, 2005.

KIESLING, T.C. Calculation of the length of the day. Agronomy Journal, Madison, v.74, p. 758-759, 1982.

LEVY, D.; KEDAR, N. Solanum tuberosum. In: HALEVY, A. H. (Ed.). CRC Handbook of flowering. Boca Raton: CRC, 1985. IV.p.363-366.
LONG, S.P. Modification of the response of photosynthetic productivity to rising temperature by atmospheric CO2 concentrations: Has its importance been underestimated? Plant, Cell and Environment, Oxford, v.14, n.6, p.729-739, 1991.

LOPES, C.A.; BUSO, J.A. Cultivo da batata (Solanum tubersoum L.). Brasília: Embrapa Hortaliças, 1997. 36p. (Instruções técnicas n. 8)

MAHLMAN, J.D. Uncertainties in projections of human induced climate warmings. Science, Washington, v.278, n.5342, p. 1416-1417, 1997.

MANRIQUE, L.A.; HODGES, T. Estimation of tuber initiation in potatoes grown in tropical environments based on different methods of computing thermal time. American Potato Journal, Orono, v.66, p.425-436, 1989.

McMASTER, G.S.; WILHELM, W.W. Growing degree-days: one equation, two interpretations. Agricultural and Forest Meteorology, Amsterdam, v.87, p.291-300, 1997.

MORENO, J.A. Clima do Rio Grande do Sul. Porto Alegre: Secretaria da Agricultura, 1961. 46p.

MUllins, E.; MILBOURNE, D.; PETTI, C.; DOYLEPRESTWICH, B.M.; MEADE, C. Potato in the age of biotechnology. Trends in Plant Science, Amsterdam, v.11, n.5, p.254-260, 2006.

NIVAA. Catálogo holandês de variedades de batata. Haarlem: Boom-Planeta, 1997. 270 p.

NYENDE, A.B.; SCHITTENHELM, S.; MIX-WAGNER, G.; GREEF, J.M. Yield and canopy development of field grown potato plants derived from synthetic seeds. European Journal of Agronomy, Amsterdam, v.22, p.175-184, 2005.

PAULA, F.L.M. Modelagem do desenvolvimento da batata (Solanum tuberosum L.) a campo. Santa Maria, 2005.42p. Dissertação (Mestrado em Agronomia) - Programa de Pós-graduação em Agronomia, Universidade Federal de Santa Maria.

PAULA, F.L.M.; STRECK, N.A.; HELDWEIN, A.B.; BISOGNIN, D.A.; PAULA, A.L.; DELLAI. J. Soma térmica de algumas fases do ciclo de desenvolvimento da batata (Solanum tuberosum L.). Ciência Rural, Santa Maria, v.35, n.5, p.1034-1042, 2005.

PENNING DE VRIES, F.W.T.;JANSEN, D.M.; TEN BERGE,H.F.M.; BAKEMA, A. Simulation of ecophysiological processes of growth in several annual crops. Wageningen: Pudok, 1989. 271p.

RICHTER, G.M.; SEMENOV, M.A. Modelling impacts of climate change on wheat yields in England and Wales: assessing drought risks. Agricultural Systems, v.84, n.1, p.77-97, 2005.

ROSENBERG, N.J.; BLAD, B.L.; VERMA, S.B. Microclimate: The biological environment. New York: John Wiley \& Sons, 1983. $495 \mathrm{p}$.

SANDS, P.J.; HACKETT, C.; NIX, H.A. A model of the development and bulking of potatoes (Solanum tuberosum L.). I - Derivation from well-managed field crops. Field Crops Research, Amsterdam, v.2, n.4, p.309-331, 1979. 
SEMENOV, M.A.; BARROW, E.M. Use of stochastic weather generator in the development of climate change scenarios. Climate Research, London, v.35, n.4, p.397-414, 1997.

SEMENOV, M.A.; BROOKS, R.J.; BARROW, E.M. Comparison of the WGEN and LARS-WG stochastic weather generators for diverse climates. Climate Research, London, v.10, n.1, p.95107, 1998.

SHAYKEWICH, C.F. An appraisal of cereal crop phenology modeling. Canadian Journal of Plant Science, Ottawa, v.75, n.2, p.329-341, 1995.

SIONIT, N.; STRAIN, B.R.; FLINT, E.P. Interaction of temperature and $\mathrm{CO} 2$ enrichment on soybean: photosynthesis and seed yield. Canadian Journal of Plant Science, Ottawa, v.67, n.5, p.629-636, 1987.

SIQUEIRA, O.J.F.; FARIAS, J.R.B.;SANS, L.M.A. Potential effects of global climate changes for Brazilian agriculture and adaptative strategies for wheat, maize and soybean. Revista Brasileira de Agrometeorologia, Santa Maria, v.2, n.1, p.115129, 1994.

SIQUEIRA, O.J.W.; STEINMETZ, S.; FERREIRA, M.F.; COSTA, A. C.; WOZNIAK, M. A. Mudanças climáticas projetadas através dos modelos GISS e reflexos na produção agrícola brasileira. Revista Brasileira de Agrometeorologia, Santa Maria, v.8, n.2, p.311-320, 2000.

SIQUEIRA, O.J.W.; STEINMETZ, S.; SALLES, L.A.B. Efeitos potenciais das mudanças climáticas na agricultura brasileira e estratégias adaptativas para algumas culturas. In: LIMA, M.A.; CABRAL, O.M.R.; MIGUEZ, J.D.G. (Eds.). Mudanças climáticas globais e a agropecuária brasileira. EMBRAPA: Jaguariúna, SP, 2001. p.33-63.

SOMBROEK, W.G.; GOMES, R. The climate change-Agriculture conundrum. In: BAZZAZ, F.; SOMBROEK, W. (Eds.). Global climate change and agricultural production. New York: John Wiley \& Sons, 1996. Cap. 1, p.1-14.

SOUZA, Z.S. Ecofisiologia. In: PEREIRA, S.A.; DANIELS, J.O. (Ed.). O cultivo da batata na Região Sul do Brasil. Brasília: Embrapa, 2003. p.80-104.

STRECK, N.A. A generalized nonlinear air temperature response function for node appearance rate in muskmelon (Cucumis melo L.). Revista Brasileira de Agrometeorologia, Santa Maria, v.10, n.1, p.105-111, 2002.

STRECK, N.A.; WEISS, A.; XUE, Q.; BAENZIGER, P. S. Improving predictions of developmental stages in winter wheat: A modified Wang and Engel model. Agricultural and Forest Meteorology, Amsterdam, v.115, n.3-4, p.139-150, 2003.

STRECK, N.A. A temperature response function for development of the chrysanthemum (Crysantemum $x$ morifolium Ramat.). Ciência Rural, Santa Maria, v.34, n.1, p.49-54, 2004.

STRECK, N.A. Climate change and agroecosystems: the effect of elevated CO2 and temperature on crop growth, development, and yield. Ciência Rural, Santa Maria, v.35, n.3, p.730-740, 2005.
STRECK, N.A.; ALBERTO, C.M. Simulação do impacto da mudança climática sobre a água disponível do solo em agroecossistemas de trigo, soja e milho em Santa Maria, RS. Ciência Rural, Santa Maria, v.36, n.2, p.424-433, 2006.

TAIZ, L.; ZEIGER, E. Fisiologia Vegetal. 3. ed. Porto Alegre: Artemed, 2004. 719p.

TUBIELLO, F.N.; DANATELLI, M.; ROSENZWEIG;STOCKLE, C.O. Effects of climate change and elevated $\mathrm{CO} 2$ on cropping systems: model predictions at two Italian locations. European Journal of Agronomy, Amsterdam, v.13, p. 179-189, 2000.

UNSWORTH, M.H.; HOGSETT, W.E. Combined effect of changing $\mathrm{CO} 2$, temperature, $\mathrm{UVB}$ radiation, and $\mathrm{O} 3$ on crop growth. In: F. BAZZAZ ; SOMBROEK, W. (Eds.) Global climate change and agricultural production. New York: John Wiley \& Sons, 1996. Cap. 8, p.171-198.

VU, J.C.V.; ALLEN, L.H.; BOOTE, K.J.; BOWES, G. Effects of elevated $\mathrm{CO} 2$ and temperature on photosynthesis and Rubisco in rice and soybean. Plant, Cell and Environment, Oxford, v.20, n.1, p.68-76, 1997.

WANG, E.; ENGEL, T. Simulation of phenological development of wheat crops. Agricultural Systems, London, v.58, n.1, p.1-24, 1998.

WIEGAND, C.L.; CUELLAR, J.A. Duration of grain filling and kernel weight of wheat as affected by temperature. Crop Science, Madison, v.21, n.1, p.95-101, 1981.

WEISS, A.; HAYS, C.J.; WON, J. Assessing winter wheat response to climate change scenarios: a simulation study in the U.S. Great Plains. Climatic Change, Wageningen, v.58, p.119-147, 2003.

WESTERMANN, D.T.; KLEINKOPF, G.E. Nitrogen requirements of potato. Agronomy Journal, Madison, v.77, p.616-621, 1985. 